\title{
Circadian rhythm disorders among adolescents: assessment and treatment options
}

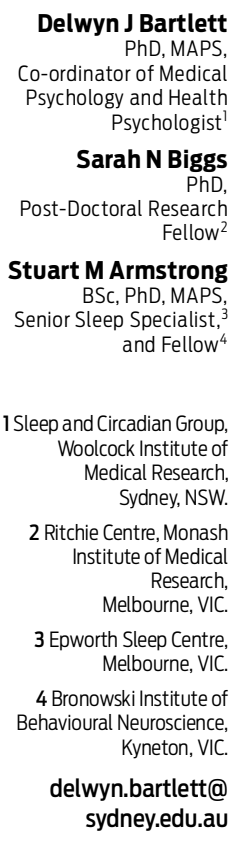

MJA 2013; 199: S16-S20 doi: $10.5694 / \mathrm{mjal3} .10912$

Online first 17/10/13
Lethargics are to be laid in the light, and exposed to the rays of the sun for the disease is gloom.

Aretaeus of Cappadocia, celebrated Greek physician, 1st century CE

\section{Circadian rhythm and the biological clock}

Biologically, the timing and duration of sleep are regulated by two interacting systems - the homoeostatic sleep drive (process S) and the circadian system (process C). ${ }^{1}$ Process $\mathrm{S}$ assumes that the longer one stays awake, the more pressure there is to fall asleep. Once asleep, this pressure dissipates until a homoeostatic equilibrium is achieved. Process $C$ regulates the timing of sleep by controlling periods of biological activity and inactivity throughout the day. These peaks and troughs in biological functioning are known as circadian rhythms and run for slightly longer than 24 hours in humans. ${ }^{2}$ Circadian rhythms are generated by the central nervous system pacemaker, the hypothalamic suprachiasmatic nucleus (SCN), sometimes called the body clock. The SCN regulates the rhythmicity of many biological processes, such as temperature and hormone release, and is responsible for synchronising these processes to each other and to the external environment. ${ }^{3}$ For all terrestrial vertebrates, evening light phase delays and morning light phase advances the biological clock. This daily resetting is how the SCN is synchronised to the 24-hour light-dark cycle and to a multitude of internal rhythms at the level of organs, tissues, cells and genes. In regard to the sleep-wake cycle, the SCN uses external cues such as light, activity and food intake (in some species) to synchronise the timing of sleep to the 24hour cycle of the social environment. Misalignment between the circadian system and the external environment, where sleep occurs outside societal norms, leads to a circadian rhythm sleep disorder. Only delayed sleep phase disorder (DSPD) and advanced sleep phase disorder are discussed in this article; other circadian rhythm sleep disorders are described elsewhere. ${ }^{4}$

\section{Common circadian rhythm sleep disorders}

DSPD is commonly found in teenagers and young adults (average age of onset, 20 years), with the pattern developing in adolescence. ${ }^{4,5}$ Sleep onset is delayed by 3-6 hours compared with conventional times (10-11 pm). ${ }^{6}$ Once sleep is attained, it is normal in length and quality but is delayed, resulting in social and often psychological difficulties. DSPD develops due to an interaction of a delay in the intrinsic circadian rhythm and poor sleep hygiene (staying up increasingly late and often using social networking).

\section{Summars \\ - Delayed sleep phase disorder (DSPD) - a circadian rhythm sleep disorder - is most commonly seen in adolescents. \\ - The differential diagnosis between DSPD and conventional psychophysiological insomnia is important for correct therapeutic intervention. \\ - Adolescent DSPD sleep duration is commonly 9 hours or more. \\ - Depression may be comorbid with DSPD. \\ - DSPD has a negative impact on adolescent academic performance. \\ - DSPD treatments include bright light therapy, chronotherapeutic regimens, and administration of melatonin as a chronobiotic (as distinct from a soporific). \\ - Attention to non-photic and extrinsic factors including healthy sleep parameters is also important to enable better sleep and mood outcomes in adolescents.}

Non-24-hour sleep-wake syndrome (also known as free-running disorder) is where the circadian clock loses synchrony to the day-night cycle and free runs, with sleep onset and wake times occurring progressively later each day. Social and environmental time cues are essentially ineffective and the pattern temporarily moves in and out of phase with societal norms. Sleep onset times may be shifted by 7 hours or more across a week. DSPD is uncommon in the general population but is found in people who are visually impaired, former rotating shift workers and some chronic fatigue/fibromyalgia sufferers.

Advanced sleep phase disorder is uncommon in adolescence, although it may manifest secondary to anxiety and depression. Sleep onset occurs early in the evening (7$9 \mathrm{pm})$, despite efforts to achieve a later bedtime. Sleep quality is typically normal but duration is often curtailed as a result of early morning waking (2-5 am). Staying in bed until the desired waking time will fragment sleep and may be misdiagnosed as irregular sleep-wake pattern.

\section{Presentation of DSPD}

DSPD is relatively common in adolescents and young adults, with a prevalence of $7 \%-16 \%$, and represents $10 \%$ of individuals diagnosed with chronic insomnia disorder in sleep clinics. ${ }^{4}$ Individuals with DSPD may have an extended circadian cycle of 24.75 hours or longer. ${ }^{3}$ The major sleep period is therefore delayed, with wake times set intractably late, leading to a propensity to fall asleep later and get up later until there is relative pattern.

When forced to be out of bed at conventional wake-up times, adolescents with DSPD continually experience a short sleep duration and feel permanently jetlagged. This may mask the true nature of the problem, resulting in a 
1 Tips for assessing and treating delayed sleep phase disorder (DSPD) in adolescents presenting with severe sleep onset insomnia

- Establish the patient's full family history - ask about sleep onset difficulties in other family members

- Establish whether there is a history of sleep onset difficulties as a child/adolescent. Is there a history of napping after school and difficulty getting up for school in the morning?

- Establish a DSPD diagnosis based on a 2-week diary in the form of a raster plot or actigraphy

- Refer the patient to a sleep clinic with circadian rhythm specialists where possible

Refer to a good reference manual - eg, Wirz-Justice et al ${ }^{15}$

- Consider a chronotherapeutic regimen for school holidays if there is considerable family support

- Establish possible core temperature minimum (2-2.5 $\mathrm{h}$ before most usual getting up time)

- Encourage light exposure (outside or artificial light for at least $40 \mathrm{~min}$ ) after the minimum core temperature time

Consider carefully timed administration of a low dose of melatonin at $1 \mathrm{mg} 4-6$ hours before prescribed bedtimes

- Once desired sleep onset time is established, maintain a dose of $0.5 \mathrm{mg}$ of melatonin $2 \mathrm{~h}$ before expected sleep onset

- Have realistic expectations - an individual successfully treated for DSPD is still likely to prefer a later sleep onset time

diagnosis of psychophysiological insomnia (PPI; also known as sleep-onset insomnia) rather than a circadian rhythm sleep disorder. Adolescents may present to a general practitioner with a history of taking "hours" to get to sleep and being extremely difficult to wake in the morning for school, university or work. They are usually accompanied by a very frustrated parent who may also describe himself or herself as a "night owl". Exploring family history is important. Adolescents may be withdrawn, indicating an underlying depression often comorbid with DSPD. ${ }^{7}$ Anxiety symptoms may also be present. The refusal to go to bed when the rest of the family do may be misinterpreted as an adolescent behavioural issue and not a genuine sleep problem. Misunderstandings from both perspectives will negatively impact on family dynamics.

An interaction between PPI and DSPD is not uncommon in adolescence, often stemming from unrealistic parental expectations. Expecting adolescents to fall asleep immediately after being mentally active with homework in the bedroom is unrealistic. The bed in that room has become a psychological reinforcement associated with heightened mental arousal and not sleeping. Time spent on the computer in the bedroom late in the evening playing video games and social messaging has a potentially similar outcome. ${ }^{8}$

Research indicates that mean optimal daytime alertness in adolescents requires a 9-hour sleep. ${ }^{9}$ This is rarely achieved, with most students cumulatively sleep-deprived as school weekdays progress, ${ }^{10}$ negatively impacting on academic performance and psychological health, ${ }^{11}$ with the added potential of motor vehicle accidents in teenage drivers. ${ }^{12}$ Restoring the correct timing, enabling sleep for daytime functioning and safety, is paramount.

\section{Treatment of DSPD}

There is a paucity of studies examining treatment of DSPD. Few have examined combinations of treatments, and some have focused only on the effects of manipulating sleep timing in healthy sleepers. ${ }^{13,14}$
DSPD may be treated by:

- a chronotherapeutic regimen: changing the timing of sleep onset to progressively delay (send forward) sleep onset until it matches a more conventional time;

- photic factors: bright light therapy;

- chronobiotic administration: use of a phase-shifter such as melatonin;

- non-photic factors and healthy sleep parameters: timing of exercise; diet; limiting the use of social media; improving mood.

Tips for assessing and treating DSPD in adolescents are provided in Box 1 .

\section{Chronotherapeutic regimen}

A raster plot (a graphic representation of sleep-wake patterns) or actigraphy (using a device resembling a wristwatch, which measures movement via an accelerometer to infer sleep/wakefulness from rest/activity cycles) are essential for recording sleep patterns over time. ${ }^{16}$ Once the current delayed sleep times are established, sleep/bedtime is progressively delayed (moved later and later), usually by 3 hours every 2 days or longer, until sleep onset time moves around the clock to reach the desired bedtime (around 10-11.30 pm). ${ }^{6}$ Exposure to post-sleep morning light (natural or artificial or a combination) is used to anchor sleep phase to the new, desired time. Sleep and temperature need to be in tandem to maintain this new desired sleep time (Box 2). This is a difficult treatment to implement, as it requires considerable planning, time away from usual daytime activities, specialist input and considerable family support.

\section{Bright light therapy}

For the whole of the animal kingdom, irrespective of whether the species is nocturnally or diurnally active, evening light exposure delays the clock while morning light phase advances it. Bright light therapy for DSPD must always be given after the core temperature minimum, which occurs 2-3 hours before wake-up time (Box 2). The body clock is then reset every day. At certain latitudes and seasons, natural exposure to dawn/dusk sunlight is not available and bright artificial light can be substituted to maintain a normal circadian phase. Bright light therapy at the appropriate post-sleep phase drives the sleeping times earlier, back to the desired bedtime (Box 3, B). Light intensity, spectrum, duration and distance from the source are crucial variables. Studies have shown the light intensity required to successfully advance the circadian phase is typically between 2500 and 10000 lux. ${ }^{17}$ However, when bright light therapy is used in combination with another therapy, such as cognitive behaviour therapy, as little as 1000 lux exposure is successful. ${ }^{18}$ Retinal cells in the lower part of the eye sending information to the SCN are tuned to the blue-green end of the spectrum, and this wavelength appears more efficacious than full-spectrum lighting. ${ }^{19}$

\section{Melatonin}

A chronobiotic is a chemical substance capable of therapeutically re-entraining short-term dissociated or longterm desynchronised circadian rhythms, or prophylactically preventing disruption following environmental insult. ${ }^{20}$ 
2 Relationship between endogenous melatonin release, 24-hour sleep-wake cycle and core temperature

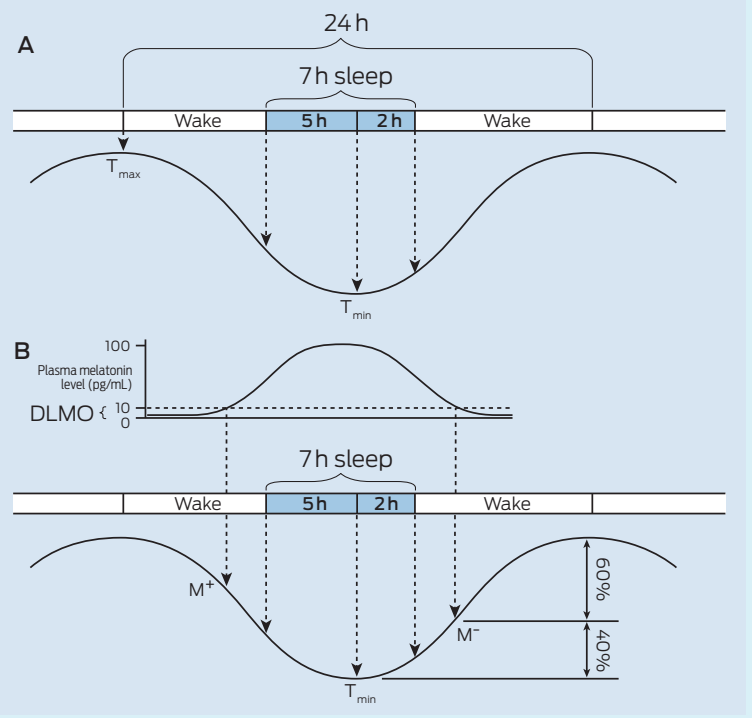

$\mathrm{DLMO}=\operatorname{dim}$ light melatonin onset. $\mathrm{M}^{+}=$melatonin onset. $\mathrm{M}^{-}=$melatonin off $\mathrm{T}_{\mathrm{max}}=$ core temperature maximum. $\mathrm{T}_{\min }=$ core temperature minimum. A. Optimal sleep onset for $7 \mathrm{~h}$ total sleep time (TST) is well down the descending limb of the core temperature rhythm and wake up time about $2-2.5 \mathrm{~h}$ after $\mathrm{T}_{\min }$. B. For an $8 \mathrm{~h}$ TST, natural wake-up time would be about $3 \mathrm{~h}$ after $\mathrm{T}_{\min }$. $\mathrm{DLMO}$ occurs about $2 \mathrm{~h}$ before sleep onset and $40 \%$ of the fall in core temperature is due to melatonin release. From the perspective of a teenager with a "normal" melatonin profile, there is no reason to expect that exogenous melatonin administration can drop core temperature any lower and thereby increase TST (in contrast to olde people with low melatonin, reduced circadian amplitude and often fragmented sleep).

Melatonin is the most researched chronobiotic in terrestrial non-seasonal breeding vertebrates. Human endogenous melatonin levels start to rise about 2 hours before natural sleep onset and peak about 5 hours later (Box 2).

About $40 \%$ of overnight core temperature decline during natural sleep is caused by the endogenous release of melatonin, which increases peripheral temperature. ${ }^{19,21}$ Time of day of melatonin administration is the critical variable with dose being second. Melatonin is administered at the reverse time of day to bright light therapy; ie, evening melatonin advances the sleep-wake cycle while evening light delays it (Box 3, A).

It is important to distinguish between the use of melatonin as a soporific (a weak hypnotic) for $\mathrm{PPI}^{15}$ and its use as a chronobiotic for treating DSPD. In the treatment of PPI, exogenous melatonin administration works best when taken 2 hours before the desired bedtime. When taken for DSPD, it may need to be administered 4-6 hours before the current sleep onset time and be moved progressively earlier as sleep onset moves earlier. ${ }^{22}$ A soporific effect may occur in the very early evening, with potential driving-safety consequences.

A combination of morning bright light therapy (after core temperature minimum) and evening melatonin can be an ideal treatment regimen. Compared with chronotherapy alone, this approach is more practical and manageable, owing to its shorter implementation period (10-20 days). ${ }^{13}$

\section{Melatonin: safety issues}

Despite assurance from studies, ${ }^{23}$ there are concerns recommending administration of high doses of melatonin.
3 Phase-response curve in relation to melatonin administration and light exposure, along with how to instigate bright light therapy

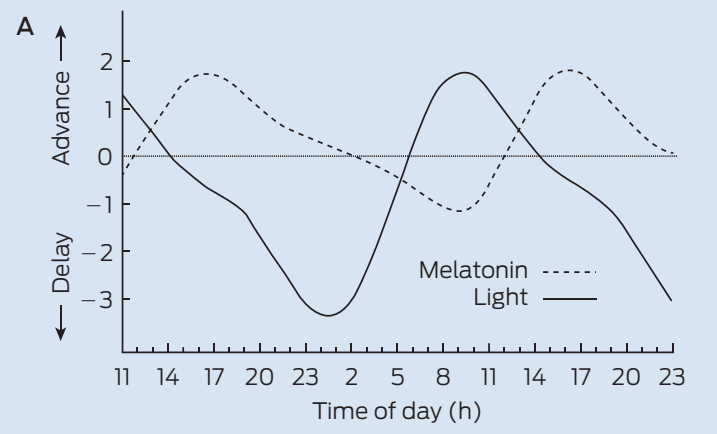

B

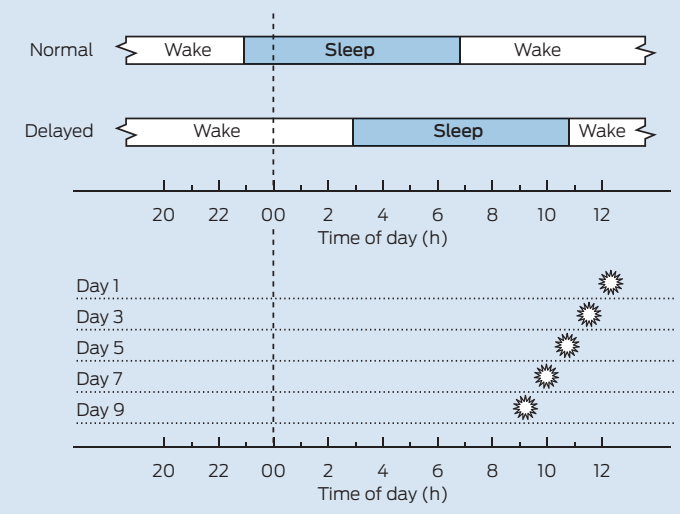

A. Phase-response curve in a normally entrained individual for melatonin ( $3 \mathrm{mg}$ ) administration over 3 consecutive days compared with bright light. Evening light phase delays the human clock while morning light phase advances. Early evening melatonin phase advances the clock while morning administration modestly delays phase. Source: Barion and Zee; ${ }^{13}$ redrawn with permission. Original data derived from Littner et $\mathrm{al}^{16}$ and Gooley. ${ }^{17}$ B. Schematic diagram of "morning" bright light therapy in a delayed sleep phase disorder patient with sleep onset at about $0300 \mathrm{~h}$ and natural wake-up time at $1100 \mathrm{~h}$. Full-spectrum bright light exposure is moved earlier and earlier every 2 days (in this example) until the target bedtime is achieved. The decision on how often to advance light exposure is made from the advancing sleep onsets recorded daily in raster plots. If pre-sleep melatonin is administered to achieve a similar result, it would be taken earlier and earlier as sleep onset advances over successive days.

Circulating endogenous melatonin levels are very high in childhood and decline precipitously at puberty, hence melatonin was speculated but not substantiated to be the pubertal hormone. ${ }^{24}$ The importance of this rapid natural decline of endogenous levels in early adolescence is unknown, and supplementing high dosages of exogenous melatonin has not been systematically researched. Although the liver is very efficient in clearing circulating levels of melatonin, with a half-life of 45-60 minutes (Box 4), a small dose of $0.3-0.5 \mathrm{mg}$ was found to be as effective as $3 \mathrm{mg}$ for advancing sleep onset. ${ }^{22,25}$ In the absence of data, the lowest effective dose of $1 \mathrm{mg}$ is recommended (compounding pharmacies).

Where sleep onset is $2 \mathrm{am}$, we suggest that melatonin be given, for example, at $8.30 \mathrm{pm}$ (ie, 5.5 hours before) for four to five nights, at $8 \mathrm{pm}$ for four to five nights, then slowly working back $(7.30 \mathrm{pm}, 7 \mathrm{pm}, 6.30 \mathrm{pm})$ until an earlier, desired sleep onset time of $11 \mathrm{pm}-12$ am is achieved. Once this sleep onset time is established, the individual can be maintained on $0.5 \mathrm{mg}$ of melatonin 2 hours before expected sleep onset (eg, 9.30-10 pm), which will then 


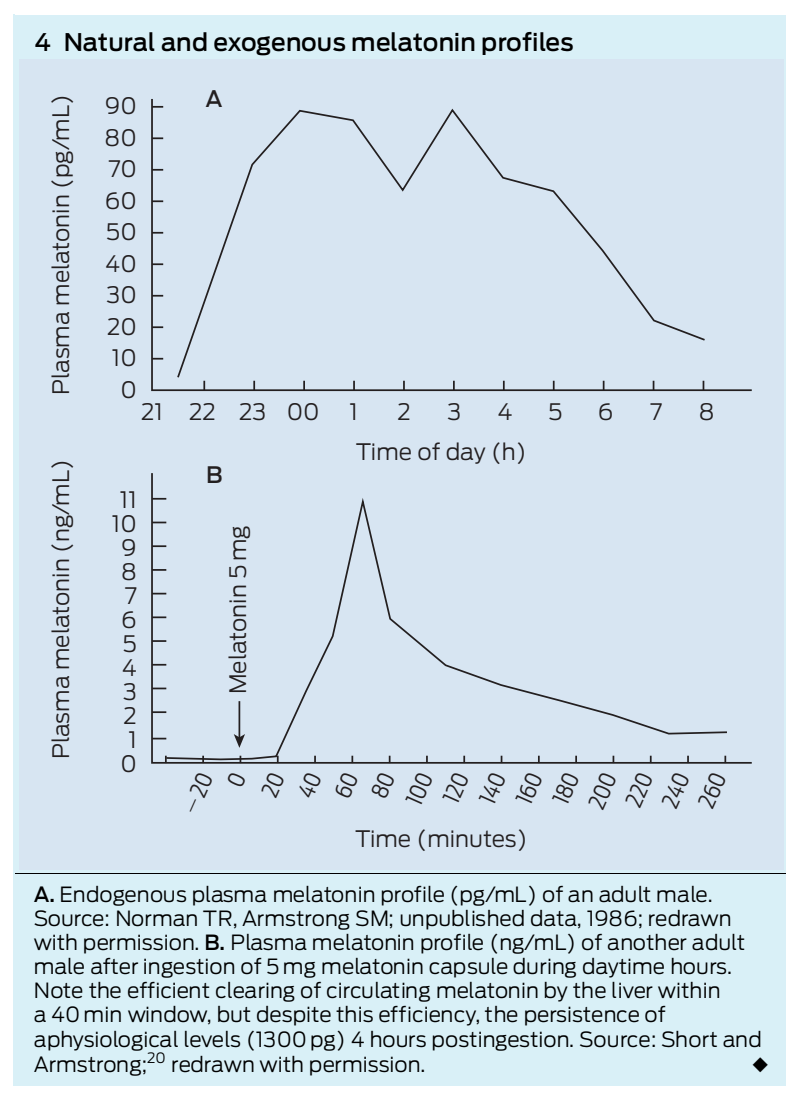

enhance the natural rise in the melatonin curve. The current prescribing norm of $3 \mathrm{mg}$ (effective for jet lag in adults) and $9 \mathrm{mg}$ doses needs more research and is not recommended for adolescents. Parental supervision is needed to ensure adherence.

Prolonged-release melatonin is thought to mimic the natural endogenous release profile, phase-advance sleep and improve sleep-maintenance insomnia when used as treatment for primary insomnia in older people (>55 years). ${ }^{26}$ Research has found the $2 \mathrm{mg}$ melatonin dose subjectively improved sleep quality and morning and evening alertness in that population. ${ }^{26}$ Anecdotally, it has been used in children and adolescents; however, until there are more research data it would be prudent not to use this medication in adolescents.

Agomelatine, currently marketed as an antidepressant, is a melatonin analogue with phase-advancing properties in rodents (as S 20098) ${ }^{27}$ and humans. ${ }^{28}$ Theoretically, agomelatine may be beneficial in older adolescents who have DSPD plus depression, since circadian changes can be associated with major depression. ${ }^{7}$ It is not the absolute delay in sleep but changes to the phase angle (timing) of sleep relative to other internal changes (onset of endogenous melatonin release relative to sleep phase) that appear crucial in the onset of depression.

\section{Non-photic and extrinsic factors}

DSPD can be exacerbated by extrinsic factors, such as use of social media (ie, electronic devices), diet, timing of exercise, and depression and anxiety. Good sleep habits or sleep hygiene are behavioural practices that result in good sleep quality and sufficient sleep duration, and prevent daytime sleepiness. ${ }^{29}$

\section{Limiting use of technology in the bedroom, particularly} in the hour before desired sleep time

The alerting effect of media is strongest when light is predominantly emitted within a blue spectrum. ${ }^{30}$ Watching television, texting and using a computer or electronic tablet device are associated with delayed sleep onset and poorer sleep quality. $8,31,32$

\section{Establishing regular sleep patterns}

Adolescents tend to sleep longer on weekends to compensate for sleep deprivation incurred over the week. If a catch-up sleep of 1-2 hours ( $9 \mathrm{am}$ ) is required, it is better for this to occur on a Saturday morning. Sunday morning get-up time needs to be $8 \mathrm{am}$, a mid point between Saturday sleep in time and the necessary Monday morning get-up time of $7 \mathrm{am}$. Some health professionals advocate adjusting the get-up time to include weekends but we believe a balance between resetting sleep and repaying sleep debt is important.

\section{Caffeine and energy-dense foods before desired sleep time}

Caffeine is a stimulant. The standard measure of one cup of espresso coffee ( $85 \mathrm{mg}$ caffeine) can last 4 hours after consumption and longer. ${ }^{33}$ Energy-dense foods, such as those high in sugar content, stimulate the digestive and endocrine system, producing an alerting effect.

\section{Exercise too close to sleep time}

In general, regular exercise is a good way to promote sleep and good health. Exercise can delay sleep in young adults if undertaken at usual sleep onset time, and prolonged aerobic exercise even a few hours earlier can maintain high body temperature, increasing alertness and interfering with evening "wind down". ${ }^{34}$

\section{Treatment for depression and anxiety}

Depression is common in DSPD. If symptoms of depression are present or develop later, it is imperative to treat to reduce exacerbation or a reduction in treatment response to DSPD. ${ }^{7}$ Sleep anxiety is commonly associated with long periods of lying in bed waiting for sleep onset in DSPD.

\section{Conclusion}

DSPD is a circadian rhythm sleep disorder that is most commonly seen in adolescents and needs to be differentiated from insomnia. Sleep diaries or actigraphy illustrating consistently delayed sleep onset and waking with normal (when unrestricted) sleep duration confirm the diagnosis. Many individuals with DSPD feel permanently jetlagged, which impacts on academic performance and has safety ramifications. Awareness and education are important components of the treatment plan, with care being taken to identify the core body temperature minimum. Without this, the effects of DSPD will be exacerbated and the individual is unlikely to respond to treatment. A combination of chronotherapeutic strategies (bright light therapy 
and melatonin) and behavioural management appears to be the most effective treatment.

Competing interests: No relevant disclosures.

Provenance: Commissioned by supplement editors; externally peer reviewed.

1 Borbély AA. A two process model of sleep regulation. Hum Neurobiol 1982; 1: 195-204.

2 Czeisler CA, Duffy JF, Shanahan TL, et al. Stability, precision, and near-24-hour period of the human circadian pacemaker. Science 1999; 284: 2177-2181.

3 Reppert SM, Weaver DR. Coordination of circadian timing in mammals. Nature 2002; 418: 935-941.

4 American Academy of Sleep Medicine. International classification of sleep disorders. Diagnostic and coding manual. 2nd ed. Westchester, Ill: AASM, 2005.

5 Wyatt J, Stepanki E, Kirby J. Circadian phase in delayed sleep phase syndrome: predictors and temporal stability across multiple assessments. Sleep 2006; 29: 1075-1080.

6 Weitzman E, Czeisler C, Coleman R, et al. Delayed sleep phase syndrome. A chronobiological disorder with sleep-onset insomnia. Arch Gen Psychiatry 1981; 38: 737-746.

7 Lewy AJ. Circadian misalignment in mood disturbances. Curr Psychiatry Rep 2009; 11: 459-465.

8 Li S, Jin X, Wu S, et al. The impact of media use on sleep patterns and sleep disorders among school-aged children in China. Sleep 2007; 30: 361-367.

9 Carskadon MA, Wolfson AR, Acebo C, et al. Adolescent sleep patterns, circadian timing, and sleepiness at a transition to early school days. Sleep 1998; 21: 871-881.

10 Carskadon MA, Harvey K, DukeP, et al. Pubertal changes in daytime sleepiness. Sleep 1980; 2: 453-460.

11 Wolfson AR, Carskadon MA. Sleep schedules and daytime functioning in adolescents. Child Dev 1998; 69: 875-887.

12 Pack Al, Pack AM, Rodgman E, et al. Characteristics of crashes attributed to the driver having fallen asleep. Accid Anal Prev 1995; 27: 769-775.

13 Barion A, Zee P. A clinical approach to circadian rhythm sleep disorders. Sleep Med 2007; 8: 566-577.

14 Burke T, Markwald R, Chinoy E, et al. Combination of light and melatonin time cues for phase advancing the human circadian clock. Sleep 2013. In press.

15 Wirz-Justice A, Benedetti F, Terman M. Chronotherapeutics for affective disorders. A clinican's manual for light and wake therapy. Basel: Karger, 2009.

16 Littner M, Kushida C, Anderson W, et al. Practice parameters for the role of actigraphy in the study of sleep and circadian rhythms: an update for 2002. Sleep 2003; 26: 337-341.

17 Gooley J. Treatment of circadian rhythm sleep disorders with light. Ann Acad Med Singapore 2008; 37: 669-676.
18 Gradisar M, Dohnt H, Gardener G, et al. A randomized controlled trial of cognitive-behavior therapy plus bright light therapy for adolescent delayed sleep phase disorder. Sleep 2011; 34: 1671-1680.

19 Wright HR, Lack LC. Effect of light wavelength on suppression and phase delay of the melatonin rhythm. Chronobiol Int 2001; 18: 801-808.

20 Short R, Armstrong S. Method for minimizing disturbances in circadian rhythms of bodily performance and function. United States Patent 4660723. 1986.

21 Krauchi K, Cajochen C, Mori D, et al. Early evening melatonin and S-20098 advance circadian phase and nocturnal regulation of body temperature. Am J Physiol 1997; 272: R1178-R1188.

22 Mundey K, Benloucif S, Harsanyi K, et al. Phase-dependent treatment of delayed sleep phase syndrome with melatonin. Sleep 2005; 28: 1271-1278.

23 Hoebert M, van der Heijden KB, van Geijlswijk IM, Smits MG. Long-term followup of melatonin treatment in children with ADHD and chronic sleep onset insomnia. J Pineal Res 2009; 47: 1-7.

24 Waldhauser $F$, Weiszenbacher $\mathrm{G}$, Frisch $\mathrm{H}$, et al. Fall in nocturnal serum melatonin during prepuberty and pubescence. Lancet 1984; 1: 362-365.

25 Burgess H, Revell V, Molina T, Eastman C. Human phase response curves to three days of daily melatonin: $0.5 \mathrm{mg}$ versus $3 \mathrm{mg}$. J Clin Endocrinol Metab 2010; 95: 3325-3331.

26 Lemoine P, Nir T, Laudon M, Zisapel N. Prolonged-release melatonin improves sleep quality and morning alertness in insomnia patients aged 55 years and older and has no withdrawal effects. J Sleep Res 2007; 16: 372-380.

27 Armstrong SM, McNulty OM, Guardiola-Lemaitre B, Redman JR. Successful use of S20098 and melatonin in an animal model of delayed sleep-phase syndrome (DSPS). Pharmacol Biochem Behav 1993; 46: 45-49.

28 Ferguson SA, Rajaratnam SM, Dawson D. Melatonin agonists and insomnia. Expert Rev Neurother 2010; 10: 305-318.

29 Mindell JA, Meltzer LJ, Carskadon MA, Chervin RD. Developmental aspects of sleep hygiene: findings from the 2004 National Sleep Foundation Sleep in America Poll. Sleep Med 2009; 10: 771-779.

30 Ruger M, St Hilaire MA, Brainard GC, et al. Human phase response curve to a single 6.5 h pulse of short-wavelength light. J Physiol 2013; 591 (Pt 1): 353-363.

31 Van den Bulck J. Text messaging as a cause of sleep interruption in adolescents, evidence from a cross-sectional study. J Sleep Res 2003; 12: 263.

32 Van den Bulck J. Television viewing, computer game playing, and Internet use and self-reported time to bed and time out of bed in secondary-school children. Sleep 2004; 27: 101-104.

33 Kamimori GH, Karyekar CS, Otterstetter R, et al. The rate of absorption and relative bioavailability of caffeine administered in chewing gum versus capsules to normal healthy volunteers. Int J Pharm 2002; 234: 159-167.

34 Baehr EK, Eastman Cl, Revelle W, et al. Circadian phase-shifting effects of nocturnal exercise in older compared with young adults. Am J Physiol 2003; 284: R1542-R1550. 